\title{
Spatiotemporal Dynamics of an HIV Infection Model with Delay in Immune Response Activation
}

\author{
Mehdi Maziane $\mathbb{D},{ }^{1}$ Khalid Hattaf $\left(\mathbb{D},{ }^{1,2}\right.$ and Noura Yousfi $\mathbb{D}^{1}$ \\ ${ }^{1}$ Department of Mathematics and Computer Science, Faculty of Sciences Ben M'sik, Hassan II University, \\ P.O. Box 7955 Sidi Othman, Casablanca, Morocco \\ ${ }^{2}$ Centre Régional des Métiers de l'Education et de la Formation (CRMEF), Derb Ghalef, 20340 Casablanca, Morocco
}

Correspondence should be addressed to Mehdi Maziane; mehdimaziane@gmail.com

Received 20 August 2017; Accepted 31 January 2018; Published 1 March 2018

Academic Editor: Peiguang Wang

Copyright (C) 2018 Mehdi Maziane et al. This is an open access article distributed under the Creative Commons Attribution License, which permits unrestricted use, distribution, and reproduction in any medium, provided the original work is properly cited.

We propose and analyse an human immunodeficiency virus (HIV) infection model with spatial diffusion and delay in the immune response activation. In the proposed model, the immune response is presented by the cytotoxic T lymphocytes (CTL) cells. We first prove that the model is well-posed by showing the global existence, positivity, and boundedness of solutions. The model has three equilibria, namely, the free-infection equilibrium, the immune-free infection equilibrium, and the chronic infection equilibrium. The global stability of the first two equilibria is fully characterized by two threshold parameters that are the basic reproduction number $R_{0}$ and the CTL immune response reproduction number $R_{1}$. The stability of the last equilibrium depends on $R_{0}$ and $R_{1}$ as well as time delay $\tau$ in the CTL activation. We prove that the chronic infection equilibrium is locally asymptotically stable when the time delay is sufficiently small, while it loses its stability and a Hopf bifurcation occurs when $\tau$ passes through a certain critical value.

\section{Introduction}

$\mathrm{HIV}$ is a virus that attacks the $\mathrm{CD} 4^{+} \mathrm{T}$ cells and reduces their number in the body. It is known that when the number of these cells is less than 200 cells per $\mu$ l, the patient enters the phase of acquired immunodeficiency syndrome (AIDS). This phase is characterized by the appearance of opportunistic infections caused by bacteria, viruses, or fungi or by the appearance of certain types of cancer. From the world health organization (WHO) [1], HIV continues to be a major global public health issue, having claimed more than 35 million lives so far. In 2016, 1 million people died from HIV-related causes globally. Also, there were approximately 36.7 million people living with HIV at the end of 2016 with 1.8 million people becoming newly infected in 2016 globally. Therefore, many mathematical models have been developed to better understand the dynamics of HIV infection. One of the earliest of these models was presented by Nowak and Bangham [2] that considers three populations: uninfected target cells, productive infected cells, and free viral particles. Rong et al. [3] extended the model of [2] by including the infected cells in eclipse stage (unproductive infected cells) and considered that a portion of these cells returns to the uninfected state.
In 2014, $\mathrm{Hu}$ et al. [4] replaced the bilinear incidence rate in [3] by a saturated infection rate and they studied the global stability of equilibria. In 2015, Maziane et al. [5] improved the model of [4] by considering the Hattaf's incidence rate [6] that includes the common types such as the bilinear incidence rate, the saturated incidence rate, the Beddington-DeAngelis functional response $[7,8]$ and the Crowley-Martin functional response [9].

Cytotoxic T lymphocytes (CTL) cells are responsible for cellular immunity and they play an important role in antiviral defense by killing the productive infected cells. For this, Lv et al. [10] proposed an HIV model with Beddington-DeAngelis functional response and CTL immune response. In 2016, Maziane et al. [11] generalized and extended the model of Lv et al. [10] by considering the mobility of cells and virus. They assumed that the motion of virus follows the Fickian diffusion and proposed the following model:

$$
\begin{aligned}
\frac{\partial T}{\partial t}= & \lambda-\mu_{T} T(x, t)-f(T(x, t), V(x, t)) V(x, t) \\
& +\rho E(x, t), \\
\frac{\partial E}{\partial t}= & f(T(x, t), V(x, t)) V(x, t)
\end{aligned}
$$




$$
\begin{gathered}
\quad-\left(\mu_{E}+\rho+\gamma\right) E(x, t), \\
\frac{\partial I}{\partial t}=\gamma E(x, t)-\mu_{I} I(x, t)-p I(x, t) C(x, t), \\
\frac{\partial V}{\partial t}=d \Delta V(x, t)+k I(x, t)-\mu_{V} V(x, t), \\
\frac{\partial C}{\partial t}=a I(x, t) C(x, t)-\mu_{C} C(x, t),
\end{gathered}
$$

where $T(x, t), E(x, t), I(x, t), V(x, t)$, and $C(x, t)$ represent the densities of uninfected $\mathrm{CD} 4^{+} \mathrm{T}$ cells, unproductive infected cells, productive infected cells, and free virus particles and CTL cells at location $x$ and time $t$, respectively. The positive parameters $\lambda, \gamma, k$, and $a$ are the production rate of uninfected cells, the rate at which infected cells in the eclipse stage become productive infected cells, the production rate of virions by infected cells, and the proliferation rate of CTL cells, respectively. The positive constants $\mu_{T}, \mu_{E}, \mu_{I}, \mu_{V}$, and $\mu_{\mathrm{C}}$ are, respectively, the death rates of uninfected $\mathrm{CD} 4^{+} \mathrm{T}$ cells, unproductive infected cells, productive infected cells, free virus, and CTL cells. The unproductive infected cells return to the uninfected cells at rate $\rho$ while the productive infected cells are killed by CTL at rate $p$. In model (1), the infection transmission process is modeled by Hattaf's incidence rate [6] of the form $f(T, V)=\beta T /\left(1+\alpha_{1} T+\right.$ $\left.\alpha_{2} V+\alpha_{3} T V\right)$, where $\alpha_{1}, \alpha_{2}$, and $\alpha_{3} \geq 0$ are the saturation factors measuring the psychological or inhibitory effect and $\beta>0$ is the infection coefficient. Here $\Delta=\sum_{i=1}^{n}\left(\partial^{2} / \partial x_{i}^{2}\right)$ is the Laplacian operator and $d$ is the diffusion coefficient of virus.

In the reality, the activation of the immune response is not instantaneous. When the virus invades the body, the immune system takes time to recognize and react to the virus. Therefore, system (1) becomes

$$
\begin{aligned}
\frac{\partial T}{\partial t}= & \lambda-\mu_{T} T(x, t) \\
& -f(T(x, t), V(x, t)) V(x, t)+\rho E(x, t), \\
\frac{\partial E}{\partial t}= & f(T(x, t), V(x, t)) V(x, t) \\
& -\left(\mu_{E}+\rho+\gamma\right) E(x, t), \\
\frac{\partial I}{\partial t}= & \gamma E(x, t)-\mu_{I} I(x, t)-p I(x, t) C(x, t), \\
\frac{\partial V}{\partial t}= & d \Delta V(x, t)+k I(x, t)-\mu_{V} V(x, t), \\
\frac{\partial C}{\partial t}= & a I(x, t-\tau) C(x, t-\tau)-\mu_{C} C(x, t),
\end{aligned}
$$

where $\tau$ denotes the time needed for the activation of the CTL immune response, namely, the immunological delay. The other parameters have the same biological meaning as system (1). In addition, we consider our model (2) with homogenous Neumann boundary condition

$$
\frac{\partial V}{\partial \nu}=0 \quad \text { on } \partial \Omega \times(0,+\infty)
$$

and initial conditions

$$
\begin{aligned}
& T(x, \theta)=\phi_{1}(x, \theta) \geq 0, \\
& E(x, \theta)=\phi_{2}(x, \theta) \geq 0,
\end{aligned}
$$

$$
\begin{gathered}
I(x, \theta)=\phi_{3}(x, \theta) \geq 0, \\
V(x, \theta)=\phi_{4}(x, \theta) \geq 0, \\
C(x, \theta)=\phi_{5}(x, \theta) \geq 0,
\end{gathered}
$$$$
x \in \bar{\Omega}, \theta \in[-\tau, 0],
$$

where $\Omega$ is a bounded domain in $\mathbb{R}^{n}$ with smooth boundary $\partial \Omega, \phi_{i}(x, \theta)(i=1,2,3,4,5)$ is Hölder continuous in $\bar{\Omega} \times$ $[-\tau, 0]$, and $\partial V / \partial \nu$ is the outward normal derivative on $\partial \Omega$.

The rest of the paper is outlined as follows. In the next section we investigate the well-posedness and equilibria for system (2)-(4). The stability analysis and the existence of Hopf bifurcation are studied in Section 3. Finally, a brief conclusion is given in Section 4.

\section{Well-Posedness and Equilibria}

In this section, we establish the existence, positivity, and boundedness of solutions of problem (2)-(4) because this model describes the evolution of a cell population. Hence the densities of cells should remain nonnegative and bounded. In addition, we determine the basic reproduction number, the CTL immune response reproduction number, and equilibria of the model (2)-(4).

Before proceeding, we shall set some notations and terminology. $X$ will denote a Banach space over a real or complex field. $C=C([-\tau, 0], X)$ will denote the Banach space of $X$-valued functions on $[-\tau, 0]$, with supremum norm, where $\tau>0$. Here, $X=C\left(\bar{\Omega}, \mathbb{R}^{5}\right)$. If $u$ is a continuous function from $[-\tau, b]$ to $X$ and $t \in[0, b]$, then $u_{t}$ denotes the element of $C$ given by $u_{t}(\theta)=u(t+\theta),-\tau \leq \theta \leq 0$.

Proposition 1. For any initial conditions satisfying (4), there exists a unique solution of problem (2)-(4) defined on $[0,+\infty)$ and this solution remains nonnegative and bounded for all $t \geq$ 0 .

Proof. Let $\phi=\left(\phi_{1}, \phi_{2}, \phi_{3}, \phi_{4}, \phi_{5}\right)^{T} \in C$ and $x \in \bar{\Omega}$. We define $F=\left(F_{1}, F_{2}, F_{3}, F_{4}, F_{5}\right)$ by

$$
\begin{aligned}
F_{1}(\phi)(x)= & \lambda-\mu_{T} \phi_{1}(x, 0) \\
& -f\left(\phi_{1}(x, 0), \phi_{4}(x, 0)\right) \phi_{4}(x, 0) \\
& +\rho \phi_{2}(x, 0), \\
F_{2}(\phi)(x)= & f\left(\phi_{1}(x, 0), \phi_{4} x, 0\right) \phi_{4}(x, 0) \\
& -\left(\rho+\mu_{E}+\gamma\right) \phi_{2}(x, 0), \\
F_{3}(\phi)(x)= & \gamma \phi_{2}(x, 0)-\mu_{I} \phi_{3}(x, 0) \\
& -p \phi_{3}(x, 0) \phi_{5}(x, 0), \\
F_{4}(\phi)(x)= & k \phi_{3}(x, 0)-\mu_{V} \phi_{4}(x, 0), \\
F_{5}(\phi)(x)= & a \phi_{3}(x,-\tau) \phi_{5}(x,-\tau)-\mu_{C} \phi_{5}(x, 0) .
\end{aligned}
$$


Hence, system (2)-(4) can be written of the form

$$
\begin{aligned}
& u^{\prime}(t)=A u(t)+F\left(u_{t}(t)\right), \quad t>0 \\
& u(0)=\phi \in X,
\end{aligned}
$$

where $u=(T, E, I, V, C)^{T}$ and $A u(t)=(0,0,0, d \Delta V, 0)^{T}$. Obviously, $F$ is locally Lipschitz in $X$. By [12-16], we deduce that system (2) admits a unique local solution on $\left[0, T_{\max }\right)$, where $T_{\max }$ is the maximal existence time for solution of system (2). In addition, $(0,0,0,0,0)$ is a lower solution of each solution of system (2); then we deduce that $T(x, t) \geq 0$, $E(x, t) \geq 0, I(x, t) \geq 0, V(x, t) \geq 0$ and $C(x, t) \geq 0$.

Next, we prove the boundedness of solutions by considering the following function:

$$
S(x, t)=T(x, t)+E(x, t)+I(x, t)+\frac{p}{a} C(x, t+\tau) .
$$

From system (2), we obtain

$$
\begin{aligned}
\frac{\partial S(x, t)}{\partial t}= & \lambda-\mu_{T} T(x, t)-\mu_{E} E(x, t)-\mu_{I} I(x, t) \\
& -\mu_{C} \frac{p}{a} C(x, t+\tau) \leq \lambda-\mu S(x, t),
\end{aligned}
$$

where $\mu=\min \left\{\mu_{T}, \mu_{E}, \mu_{I}, \mu_{C}\right\}$. Thus,

$$
\begin{aligned}
& S(x, t) \leq \max \left\{\frac{\lambda}{\mu}, \max _{x \in \bar{\Omega}}\left\{\phi_{1}(x, 0)+\phi_{2}(x, 0)+\phi_{3}(x, 0)\right.\right. \\
& \left.\left.+\frac{p}{a} \phi_{5}(x,-\tau)\right\}\right\} .
\end{aligned}
$$

Then, $T, E, I$, and $C$ are bounded.

To prove the boundedness of $V$, from system (2), we get

$$
\begin{aligned}
\frac{\partial V(x, t)}{\partial t}-d_{V} \Delta V & \leq k \delta-\mu_{V} V, \\
\frac{\partial V(x, t)}{\partial v} & =0, \\
V(x, 0) & =\max _{x \in \bar{\Omega}} \phi_{4}(x, 0),
\end{aligned}
$$

where $\delta=\max \left\{\lambda / \mu, \max _{x \in \bar{\Omega}}\left\{\phi_{1}(x, 0)+\phi_{2}(x, 0)+\phi_{3}(x, 0)+\right.\right.$ $\left.\left.(p / a) \phi_{5}(x,-\tau)\right\}\right\}$.

Using the comparison principle [17], we have $V(x, t) \leq$ $\bar{V}(t)$, where $\bar{V}(t)=\phi_{4}(x) e^{-\mu_{V} t}+\left(k \delta / \mu_{V}\right)\left(1-e^{-\mu_{V} t}\right)$ is the solution of the problem

$$
\begin{aligned}
\frac{d \bar{V}}{d t} & =k \delta-\mu_{V} \bar{V} \\
\bar{V}(0) & =\max _{x \in \bar{\Omega}} \phi_{4}(x, 0) .
\end{aligned}
$$

Since $\bar{V}(t) \leq \max \left\{k \delta / \mu_{V}, \max _{x \in \bar{\Omega}} \phi_{4}(x, 0)\right\}, \forall(x, t) \in \bar{\Omega} \times$ $\left[0, T_{\max }\right)$, we have that $V$ is bounded.

Therefore, we have proved that $T(x, t), E(x, t), I(x, t)$, $V(x, t)$, and $C(x, t)$ are bounded on $\bar{\Omega} \times\left[0, T_{\max }\right)$. Hence, it follows from the standard theory for semilinear parabolic systems [18] that $T_{\max }=+\infty$.
As in [11], the basic reproduction number of virus in the absence of spatial dependence is given by

$$
R_{0}=\frac{\lambda \beta k \gamma}{\mu_{I} \mu_{V}\left(\lambda \alpha_{1}+\mu_{T}\right)\left(\mu_{E}+\gamma\right)} .
$$

In addition to $R_{0}$, we define the CTL immune response reproduction number $R_{1}$ of our model by

$$
R_{1}=\frac{a I_{1}}{\mu_{C}},
$$

which represents the threshold level to activate the CTL cells response.

\section{Theorem 2.}

(i) If $R_{0} \leq 1$, system (2) has always an infection-free equilibrium of the form $Q_{0}\left(\lambda / \mu_{T}, 0,0,0,0\right)$.

(ii) If $R_{0}>1$, system (2) has an immune-free equilibrium of the form $Q_{1}\left(T_{1}, E_{1}, I_{1}, V_{1}, 0\right)$ with $T_{1} \in\left(0, \lambda / \mu_{T}\right)$, $E_{1} \geq 0, I_{1} \geq 0$, and $V_{1} \geq 0$.

(iii) If $R_{1}>1$, system (2) has a chronic infection equilibrium of the form $Q_{2}\left(T_{2}, E_{2}, I_{2}, V_{2}, C_{2}\right)$ with $T_{2} \in\left(0, \lambda / \mu_{T}-\right.$ $\left.\mu_{I} \mu_{C}\left(\mu_{E}+\gamma\right) / a \gamma \mu_{T}\right), E_{2} \geq 0, I_{2} \geq 0, V_{2} \geq 0$, and $\mathrm{C}_{2} \geq 0$.

\section{Stability Analysis and Hopf Bifurcation}

First, we discuss the global stability of the infection-free equilibrium $Q_{0}$ and the immune-free equilibrium $Q_{1}$.

\section{Theorem 3.}

(i) The infection-free equilibrium $Q_{0}$ is globally asymptotically stable if $R_{0} \leq 1$.

(ii) The immune-free equilibrium $Q_{1}$ is globally asymptotically stable if $R_{1} \leq 1<R_{0}$ and

$$
\begin{aligned}
R_{0} & \leq 1 \\
& +\frac{\left[\mu_{T} \mu_{I} \mu_{V}\left(\mu_{E}+\gamma\right)+\alpha_{2} \mu_{T} \lambda k \gamma\right]\left(\mu_{E}+\rho+\gamma\right)+\rho \alpha_{3} k \gamma \lambda^{2}}{\rho \mu_{I} \mu_{V}\left(\mu_{E}+\rho+\gamma\right)\left(\mu_{T}+\alpha_{1} \lambda\right)} .
\end{aligned}
$$

Proof. By using the method proposed by Hattaf and Yousfi [19], we propose the following Lyapunov functional for system (2)-(4) at $Q_{0}$ :

$$
\begin{aligned}
W_{0} & =\int_{\Omega}\left[T(x, t)-T_{0}-\int_{T_{0}}^{T(x, t)} \frac{f\left(T_{0}, 0\right)}{f(S, 0)} d S\right. \\
& +\frac{\rho\left(T(x, t)-T_{0}+E(x, t)\right)^{2}}{2\left(1+\alpha_{1} T_{0}\right)\left(\mu_{T}+\mu_{E}+\gamma\right) T_{0}}+\frac{\rho+\mu_{E}+\gamma}{\gamma} \\
& \cdot I(x, t)+E(x, t)+\frac{\mu_{I}\left(\rho+\mu_{E}+\gamma\right)}{k \gamma} V(x, t) \\
+ & \frac{p\left(\rho+\mu_{E}+\gamma\right)}{a \gamma} C(x, t)+\frac{p\left(\rho+\mu_{E}+\gamma\right)}{\gamma} \\
. & \left.\int_{t-\tau}^{t} I(x, \theta) C(x, \theta) d \theta\right] d x,
\end{aligned}
$$


where $T_{0}=\lambda / \mu_{T}$. For convenience, we let $\psi(x, t)=\psi$ and $\psi(x, t-\tau)=\psi_{\tau}$, for any $\psi \in\{T, E, I, V, C\}$. By calculation, we have

$$
\begin{aligned}
& \frac{d W_{0}}{d t}=\int_{\Omega}\left[\left(1-\frac{f\left(T_{0}, 0\right)}{f(T, 0)}\right) \frac{\partial T}{\partial t}\right. \\
& +\frac{\rho\left(T-T_{0}+E\right)(\partial T / \partial t+\partial E / \partial t)}{\left(1+\alpha_{1} T_{0}\right)\left(\mu_{T}+\mu_{E}+\gamma\right) T_{0}} \\
& +\frac{\rho+\mu_{E}+\gamma}{\gamma} \frac{\partial I}{\partial t}+\frac{\partial E}{\partial t}+\frac{\mu_{I}\left(\rho+\mu_{E}+\gamma\right)}{k \gamma} \frac{\partial V}{\partial t} \\
& +\frac{p\left(\rho+\mu_{E}+\gamma\right)}{a \gamma} \frac{\partial C}{\partial t} \\
& \left.+\frac{p\left(\rho+\mu_{E}+\gamma\right)}{\gamma} \frac{\partial}{\partial t} \int_{t-\tau}^{t} I_{\theta} C_{\theta} d \theta\right] d x .
\end{aligned}
$$

Noting that $\lambda=\mu_{T} T_{0}$, the time derivative of $W_{0}$ along the positive solutions of system (2) satisfies

$$
\begin{aligned}
& \frac{d W_{0}}{d t}=\int_{\Omega}\left[\left(1-\frac{f\left(T_{0}, 0\right)}{f(T, 0)}\right) \mu_{T}\left(T_{0}-T\right)\right. \\
& +\frac{f\left(T_{0}, 0\right) f(T, V)}{f(T, 0)} V+\rho\left(1-\frac{f\left(T_{0}, 0\right)}{f(T, 0)}\right) E \\
& -\frac{\rho \mu_{T}\left(T-T_{0}\right)^{2}}{\left(1+\alpha_{1} T_{0}\right)\left(\mu_{T}+\mu_{E}+\gamma\right) T_{0}} \\
& -\frac{\rho\left(\mu_{E}+\gamma\right) E^{2}}{\left(1+\alpha_{1} T_{0}\right)\left(\mu_{T}+\mu_{E}+\gamma\right) T_{0}} \\
& +\frac{\rho E}{\left(1+\alpha_{1} T_{0}\right) T_{0}}\left(T_{0}-T\right)-\frac{p\left(\rho+\mu_{E}+\gamma\right)}{\gamma} I C \\
& -\frac{\mu_{I} \mu_{V}\left(\rho+\mu_{E}+\gamma\right)}{k \gamma} V+\frac{p\left(\rho+\mu_{E}+\gamma\right)}{\gamma} I_{\tau} C_{\tau} \\
& -\frac{\mu_{C} p\left(\rho+\mu_{E}+\gamma\right)}{a \gamma} C \\
& +\frac{p\left(\rho+\mu_{E}+\gamma\right)}{\gamma}\left[I C-I_{\tau} C_{\tau}\right] \\
& \left.+\frac{d \mu_{I}\left(\rho+\mu_{E}+\gamma\right)}{k \gamma} \Delta V\right] d x \\
& =-\int_{\Omega}\left[\left(\frac{1}{T}+\frac{\rho}{\left(\mu_{T}+\mu_{E}+\gamma\right) T_{0}}\right) \frac{\mu_{T}\left(T-T_{0}\right)^{2}}{1+\alpha_{1} T_{0}}\right. \\
& +\frac{\rho\left(\mu_{E}+\gamma\right) E^{2}}{\left(1+\alpha_{1} T_{0}\right)\left(\mu_{T}+\mu_{E}+\gamma\right) T_{0}}+\frac{\rho\left(T-T_{0}\right)^{2} E}{\left(1+\alpha_{1} T_{0}\right) T T_{0}} \\
& -\frac{\mu_{I} \mu_{V}\left(\rho+\mu_{E}+\gamma\right)}{k \gamma}\left(R_{0}-1\right) V
\end{aligned}
$$

$$
\begin{aligned}
& +\frac{\left(\alpha_{2}+\alpha_{3} T\right) V^{2}}{1+\alpha_{1} T+\alpha_{2} V+\alpha_{3} T V} f\left(T_{0}, 0\right) \\
& \left.+\frac{\mu_{C} p\left(\rho+\mu_{E}+\gamma\right)}{a \gamma} C\right] d x .
\end{aligned}
$$

Therefore, $d W_{0} / d t \leq 0$ if $R_{0} \leq 1$. In addition, it is not hard to verify that the largest compact invariant set in $\left\{(T, E, I, V, C) \mid d W_{0} / d t=0\right\}$ is just the singleton $\left\{Q_{0}\right\}$. From LaSalle invariance principle [20], we deduce that $Q_{0}$ is globally asymptotically stable.

Next, we construct the Lyapunov functional for system (2)-(4) at $Q_{1}$ :

$$
\begin{aligned}
W_{1} & =\int_{\Omega}\left[T-T_{1}-\int_{T_{1}}^{T} \frac{f\left(T_{1}, V_{1}\right)}{f\left(S, V_{1}\right)} d S\right. \\
& +\frac{\rho\left(1+\alpha_{2} V_{1}\right)\left(T-T_{1}+E-E_{1}\right)^{2}}{2\left(1+\alpha_{1} T_{1}+\alpha_{2} V_{1}+\alpha_{3} T_{1} V_{1}\right)\left(\mu_{T}+\mu_{E}+\gamma\right) T_{1}} \\
& +\frac{f\left(T_{1}, V_{1}\right) V_{1}}{\gamma E_{1}} I_{1} \Phi\left(\frac{I}{I_{1}}\right)+E_{1} \Phi\left(\frac{E}{E_{1}}\right) \\
& +\frac{\mu_{I} f\left(T_{1}, V_{1}\right) V_{1}}{k \gamma E_{1}} V_{1} \Phi\left(\frac{V}{V_{1}}\right)+\frac{p f\left(T_{1}, V_{1}\right) V_{1}}{a \gamma E_{1}} C \\
& \left.+\frac{p f\left(T_{1}, V_{1}\right) V_{1}}{\gamma E_{1}} \int_{t-\tau}^{t} I_{\theta} C_{\theta} d \theta\right] d x
\end{aligned}
$$

where $\Phi(x)=x-1-\ln (x)$. Obviously, the function $\Phi$ has a global minimum at 1 and satisfies $\Phi(1)=0$. Calculating the time derivative of $W_{1}$ along the positive solutions of system (2) and applying $\lambda=\mu_{T} T_{1}+f\left(T_{1}, V_{1}\right) V_{1}-\rho E_{1}$, we obtain

$$
\begin{aligned}
& \frac{d W_{1}}{d t}=\int_{\Omega}\left[\left(1-\frac{f\left(T_{1}, V_{1}\right)}{f\left(T, V_{1}\right)}\right) \mu_{T}\left(T_{1}-T\right)\right. \\
& +\frac{f\left(T_{1}, V_{1}\right) f(T, V)}{f\left(T, V_{1}\right)} V+\rho\left(1-\frac{f\left(T_{1}, V_{1}\right)}{f\left(T, V_{1}\right)}\right) E \\
& -\frac{\mu_{T} \rho\left(1+\alpha_{2} V_{1}\right)}{\left(1+\alpha_{1} T_{1}+\alpha_{2} V_{1}+\alpha_{3} T_{1} V_{1}\right)\left(\mu_{T}+\mu_{E}+\gamma\right) T_{1}}\left(T-T_{1}\right)^{2} \\
& -\frac{\rho\left(1+\alpha_{2} V_{1}\right)\left(\mu_{E}+\gamma\right)}{\left(1+\alpha_{1} T_{1}+\alpha_{2} V_{1}+\alpha_{3} T_{1} V_{1}\right)\left(\mu_{T}+\mu_{E}+\gamma\right) T_{1}}\left(E-E_{1}\right)^{2} \\
& -\frac{\rho\left(1+\alpha_{2} V_{1}\right)\left(E-E_{1}\right)\left(T-T_{1}\right)^{2}}{\left(1+\alpha_{1} T_{1}+\alpha_{2} V_{1}+\alpha_{3} T_{1} V_{1}\right) T_{1} T}-\frac{\left(f\left(T_{1}, V_{1}\right)\right)^{2}}{f\left(T, V_{1}\right)} V_{1} \\
& +4 f\left(T_{1}, V_{1}\right) V_{1}-f\left(T_{1}, V_{1}\right) V-f\left(T_{1}, V_{1}\right) V_{1} \frac{I_{1} E}{I E_{1}} \\
& -f(T, V) V \frac{E_{1}}{E}-f\left(T_{1}, V_{1}\right) V_{1} \frac{V_{1} I}{I_{1} V}+\frac{p f\left(T_{1}, V_{1}\right) V_{1}}{\gamma E_{1}}\left(I_{1}\right. \\
& \left.\left.-\frac{\mu_{C}}{a}\right) C\right] d x+\frac{d \mu_{I} f\left(T_{1}, V_{1}\right) V_{1}}{k \gamma E_{1}} \int_{\Omega}\left(1-\frac{V_{1}}{V}\right) \Delta V d x
\end{aligned}
$$




$$
\begin{aligned}
& =-\int_{\Omega}\left[\frac { ( 1 + \alpha _ { 2 } V _ { 1 } ) ( T - T _ { 1 } ) ^ { 2 } } { T T _ { 1 } ( 1 + \alpha _ { 1 } T _ { 1 } + \alpha _ { 2 } V _ { 1 } + \alpha _ { 3 } T _ { 1 } V _ { 1 } ) } \left(\left(\mu_{T} T_{1}-\rho E_{1}\right)\right.\right. \\
& \left.+\frac{\rho \mu_{T} T}{\mu_{T}+\mu_{E}+\gamma}+\rho E\right) \\
& +\frac{\rho\left(E-E_{1}\right)^{2}\left(1+\alpha_{2} V_{1}\right)\left(\mu_{E}+\gamma\right)}{T_{1}\left(1+\alpha_{1} T_{1}+\alpha_{2} V_{1}+\alpha_{3} T_{1} V_{1}\right)\left(\mu_{T}+\mu_{E}+\gamma\right)}-f\left(T_{1}, V_{1}\right) \\
& \cdot V_{1}\left(5-\frac{f\left(T_{1}, V_{1}\right)}{f\left(T, V_{1}\right)}-\frac{I_{1} E}{E_{1} I}-\frac{f(T, V)}{f\left(T_{1}, V_{1}\right)} \frac{V E_{1}}{V_{1} E}-\frac{V_{1} I}{V I_{1}}\right. \\
& \left.-\frac{f\left(T, V_{1}\right)}{f(T, V)}\right) \\
& +\frac{f\left(T_{1}, V_{1}\right)\left(1+\alpha_{1} T\right)\left(\alpha_{2}+\alpha_{3} T\right)\left(V-V_{1}\right)^{2}}{\left(1+\alpha_{1} T+\alpha_{2} V_{1}+\alpha_{3} T V_{1}\right)\left(1+\alpha_{1} T+\alpha_{2} V+\alpha_{3} T V\right)} \\
& \left.-\frac{p \mu_{C} f\left(T_{1}, V_{1}\right) V_{1}}{a \gamma E_{1}}\left(R_{1}-1\right) C\right] d x \\
& -\frac{d \mu_{I} f\left(T_{1}, V_{1}\right) V_{1}^{2}}{k \gamma E_{1}} \int_{\Omega} \frac{\|\nabla V\|^{2}}{V^{2}} d x .
\end{aligned}
$$

Using the arithmetic-geometric inequality, we get

$$
\begin{gathered}
5-\frac{f\left(T_{1}, V_{1}\right)}{f\left(T, V_{1}\right)}-\frac{I_{1} E}{E_{1} I}-\frac{f(T, V)}{f\left(T_{1}, V_{1}\right)} \frac{V E_{1}}{V_{1} E}-\frac{V_{1} I}{V I_{1}} \\
-\frac{f\left(T, V_{1}\right)}{f(T, V)} \leq 0 .
\end{gathered}
$$

Therefore, $d W_{1} / d t \leq 0$ if $R_{1} \leq 1$ and $\rho E_{1} \leqslant \mu_{T} T_{1}$.

Obviously, the condition $\rho E_{1} \leqslant \mu_{T} T_{1}$ is equivalent to

$$
\begin{aligned}
R_{0} & \leq 1 \\
& +\frac{\left[\mu_{T} \mu_{I} \mu_{V}\left(\mu_{E}+\gamma\right)+\alpha_{2} \mu_{T} \lambda k \gamma\right]\left(\mu_{E}+\rho+\gamma\right)+\rho \alpha_{3} k \gamma \lambda^{2}}{\rho \mu_{I} \mu_{V}\left(\mu_{E}+\rho+\gamma\right)\left(\mu_{T}+\alpha_{1} \lambda\right)} .
\end{aligned}
$$

In addition, $d W_{1} / d t=0$ if and only if $T=T_{1}, E=E_{1}, I=I_{1}$, $V=V_{1}$, and $C=0$. Hence, the largest compact invariant set in $\left\{(T, E, I, V, C) \mid d W_{1} / d t=0\right\}$ is the singleton $\left\{Q_{1}\right\}$. This proves the global stability of $Q_{1}$ by using LaSalle's invariance principle [20].

From the above theorem, we deduce that the time delay in the activation of CTL immune response has no effect on the stability of $Q_{0}$ and $Q_{1}$. Next we investigate the stability and existence of Hopf bifurcation at the chronic infection equilibrium $Q_{2}$.

When $\tau=0$, system (2) becomes system (1). By Theorem 3 (iii) [11], we deduce the following result.

Theorem 4. When $\tau=0$, the chronic infection equilibrium with immune response $Q_{2}$ is globally asymptotically stable if $R_{1}>1$ and

$$
\begin{aligned}
k \beta \mu_{C} \rho \leq & \alpha_{1} \lambda \rho a \mu_{V}+\mu_{T}\left(\rho+\mu_{E}+\gamma\right)\left(\alpha_{2} k \mu_{C}+a \mu_{V}\right) \\
& +\alpha_{3} \rho \lambda k \mu_{C} .
\end{aligned}
$$

Now, we study the existence of Hopf bifurcation by regarding time delay $\tau$ as the bifurcation parameter.

Let $0=\mu_{0}<\mu_{1}<\cdots<\mu_{n}<\cdots$ be the eigenvalues of $-\Delta$ on $\Omega$ with homogeneous Neumann boundary conditions, and for $=0,1,2, \ldots$, let $E\left(\mu_{i}\right)$ be the space of eigenfunctions corresponding to $\mu_{i}$ in $C^{1}(\Omega)$. Let $\left\{\phi_{i j}: j=1,2, \ldots, \operatorname{dim} E\left(\mu_{i}\right)\right\}$ be an orthonormal basis of $E\left(\mu_{i}\right), X=\left[C^{1}(\Omega)\right]^{5}$ and $X_{i j}=\left\{c \phi_{i j}: c \in \mathbb{R}^{5}\right\}$. Then, $X=\bigoplus_{i=1}^{\infty} X_{i}, X_{i}=\bigoplus_{j=1}^{\operatorname{dim}\left[S\left(\mu_{i}\right)\right]} X_{i j}$.

The linearization of system (2) at the constant solution $Q_{2}\left(T_{2}, E_{2}, I_{2}, V_{2}, C_{2}\right)$ can be expressed by

$$
\frac{\partial Z}{\partial t}=D \Delta Z+J Z+J^{*} Z_{\tau}
$$

where $Z=(T, E, I, V, C), Z_{\tau}=\left(T_{\tau}, E_{\tau}, I_{\tau}, V_{\tau}, C_{\tau}\right)$, and $D=$ $(0,0,0, d, 0)$,

$$
\begin{aligned}
& J=\left(\begin{array}{ccccc}
-\frac{\partial f\left(T_{2}, V_{2}\right)}{\partial T} V_{2}-\mu_{T} & \rho & -\frac{\partial f\left(T_{2}, V_{2}\right)}{\partial V} V_{2}-f\left(T_{2}, V_{2}\right) & 0 & 0 \\
\frac{\partial f\left(T_{2}, V_{2}\right)}{\partial V} V_{2} & -\left(\mu_{E}+\rho+\gamma\right) & 0 & \frac{\partial f\left(T_{2}, V_{2}\right)}{\partial V} V_{2}+f\left(T_{2}, V_{2}\right) & 0 \\
0 & \gamma & \mu_{I}-p C_{2} & 0 & -p I_{2} \\
0 & 0 & k & -\mu_{V} & 0 \\
0 & 0 & 0 & 0 & -\mu_{C}
\end{array}\right), \\
& J^{*}=\left(\begin{array}{ccccc}
0 & 0 & 0 & 0 & 0 \\
0 & 0 & 0 & 0 & 0 \\
0 & 0 & 0 & 0 & 0 \\
0 & 0 & 0 & 0 & 0 \\
0 & 0 & a C_{2} & 0 & a I_{2}
\end{array}\right)
\end{aligned}
$$


Let $\mathscr{L} Z=D \Delta Z+J Z(x, t)+J^{*} Z(x, t-\tau)$. For each $i=$ $0,1,2, \ldots, X_{i}$ is invariant under the operator $\mathscr{L}$, and $\xi$ is an eigenvalue of $\mathscr{L}$ if and only if it is satisfying the characteristic equation

$$
\operatorname{det}\left(-\xi I-\mu_{i} D+J+e^{-\xi \tau} J^{*}\right)=0 .
$$

Then, at $Q_{2}$, the associated characteristic equation of system (2) is given by

$$
\begin{aligned}
\xi^{5}+ & a_{1} \xi^{4}+a_{2} \xi^{3}+a_{3} \xi^{2}+a_{4} \xi+a_{5} \\
& +e^{-\xi \tau}\left(b_{1} \xi^{4}+b_{2} \xi^{3}+b_{3} \xi^{2}+b_{4} \xi+b_{5}\right)=0
\end{aligned}
$$

where

$$
\begin{aligned}
a_{1} & =\frac{\partial f\left(T_{2}, V_{2}\right)}{\partial T} V_{2}+\mu_{T}+\mu_{I}+\mu_{V}+\mu_{C}+p C_{2}+\mu_{E} \\
& +\rho+\gamma+\mu_{i} d, \\
a_{2} & =\left(\frac{\partial f\left(T_{2}, V_{2}\right)}{\partial T} V_{2}+\mu_{T}\right)\left[\left(\mu_{E}+\rho+\gamma\right)\right. \\
& \left.+\left(\mu_{V}+\mu_{i} d\right)+\mu_{I}+\mu_{C}+p C_{2}\right]+\left(\mu_{E}+\rho+\gamma\right)\left(\mu_{V}\right. \\
& \left.+\mu_{i} d+\mu_{I}+\mu_{C}+p C_{2}\right)+\left(\mu_{I}+p C_{2}\right)\left(\mu_{V}+\mu_{i} d\right. \\
& \left.+\mu_{C}\right)+\left(\mu_{V}+\mu_{i} d\right) \mu_{C}+\mu_{T} \rho, \\
a_{3} & =\left(\frac{\partial f\left(T_{2}, V_{2}\right)}{\partial T} V_{2}+\mu_{T}\right)\left[\left(\mu_{V}+\mu_{i} d+\mu_{C}\right)\right. \\
& \left.+\left(\mu_{I}+p C_{2}\right)+\left(\mu_{V}+\mu_{i} d\right) \mu_{C}\right]+\left(\frac{\partial f\left(T_{2}, V_{2}\right)}{\partial T} V_{2}\right. \\
& \left.+\mu_{T}\right)\left(\mu_{E}+\gamma\right)\left(\mu_{I}+\mu_{V}+\mu_{i} d+\mu_{C}+p C_{2}\right) \\
& +\mu_{C}\left(\mu_{V}+\mu_{i} d\right)\left(\mu_{I}+p C_{2}+\mu_{E}+\rho+\gamma\right) \\
& +\mu_{C}\left(\mu_{I}+p C_{2}\right)+\left(\mu_{E}+\gamma\right)\left(\mu_{V}+\mu_{i} d+\mu_{I}+p C_{2}\right) \\
& +k \gamma \frac{\partial f\left(T_{2}, V_{2}\right)}{\partial V} V_{2}+\mu_{T} \rho\left(\mu_{V}+\mu_{i} d+\mu_{I}+\mu_{C}\right. \\
a_{4} & =\left(\frac{\partial f\left(T_{2}, V_{2}\right)}{\partial T} V_{2}+\mu_{T}\right)\left[\left(\mu_{V}+\mu_{i} d\right)\right. \\
& \left.+V_{2}\right) \\
& \left.\left.+\mu_{2}\right]-\mu_{i} d\right)+\left(\mu_{V}+\mu_{i} d\right) \\
& \\
& \\
&
\end{aligned}
$$

$$
\begin{aligned}
\left.\cdot \mu_{C}\right)+\frac{\partial f\left(T_{2}, V_{2}\right)}{\partial T} V_{2}\left[k \gamma \frac{\partial f\left(T_{2}, V_{2}\right)}{\partial V} V_{2}\right. \\
\left.+\left(\mu_{V}+\mu_{i} d\right)\left(\mu_{E}+\gamma\right)\left(\mu_{I}+p C_{2}\right)\right], \\
a_{5}=-\left(\frac{\partial f\left(T_{2}, V_{2}\right)}{\partial T} V_{2}+\mu_{T}\right) k \gamma \frac{\partial f\left(T_{2}, V_{2}\right)}{\partial V} V_{2} \mu_{C} \\
-k \gamma \mu_{T}\left(\mu_{V}+\mu_{i} d\right) \frac{\partial f\left(T_{2}, V_{2}\right)}{\partial V} V_{2} \\
+\frac{\partial f\left(T_{2}, V_{2}\right)}{\partial T} V_{2} \mu_{C}\left(\frac{\partial f\left(T_{2}, V_{2}\right)}{\partial V} V_{2}+f\left(T_{2}, V_{2}\right)\right), \\
b_{1}=-\mu_{C},
\end{aligned}
$$$$
b_{2}=-\mu_{C}\left[\frac{\partial f\left(T_{2}, V_{2}\right)}{\partial T} V_{2}+\mu_{T}+\mu_{I}+\mu_{V}+\mu_{i} d+\mu_{E}\right.
$$$$
+\rho+\gamma]
$$$$
b_{3}=-\mu_{C}\left[\left(\frac{\partial f\left(T_{2}, V_{2}\right)}{\partial T} V_{2}+\mu_{T}\right)\right.
$$$$
\cdot\left(\mu_{I}+\mu_{V}+\mu_{i} d+\mu_{E}+\gamma\right)+\mu_{I}\left(\mu_{V}+\mu_{i} d\right)
$$$$
\left.+\left(\mu_{I}+\mu_{V}+\mu_{i} d\right)\left(\mu_{E}+\rho+\gamma\right)+\rho \mu_{T}\right] \text {, }
$$$$
b_{4}=-\mu_{C}\left[\left(\frac{\partial f\left(T_{2}, V_{2}\right)}{\partial T} V_{2}+\mu_{T}\right)\right.
$$$$
\cdot\left(\mu_{I}\left(\mu_{V}+\mu_{i} d\right)+\left(\mu_{E}+\gamma\right)\left(\mu_{I}+\mu_{V}+\mu_{i} d\right)\right)
$$$$
-k \gamma\left(\frac{\partial f\left(T_{2}, V_{2}\right)}{\partial V} V_{2}+f\left(T_{2}, V_{2}\right)\right)
$$$$
\left.+\mu_{T} \rho\left(\mu_{I}+\mu_{V}+\mu_{i} d\right)\right],
$$$$
b_{5}=-\mu_{C}\left[\left(\frac{\partial f\left(T_{2}, V_{2}\right)}{\partial T} V_{2}+\mu_{T}\right) \mu_{I}\left(\mu_{V}+\mu_{i} d\right)\right.
$$$$
\cdot\left(\mu_{E}+\gamma\right)-k \gamma \mu_{T}\left(\frac{\partial f\left(T_{2}, V_{2}\right)}{\partial V} V_{2}+f\left(T_{2}, V_{2}\right)\right)
$$$$
\left.+\rho \mu_{T} \mu_{I}\left(\mu_{V}+\mu_{i} d\right)\right] \text {. }
$$

For $\tau \neq 0$, we suppose that (26) has a purely imaginary root $\xi=i \omega$ with $\omega>0$. Substituting $\xi=i \omega$ in (26) and separating the real and the imaginary parts, we get

$$
\begin{aligned}
w^{5}-a_{2} w^{3}+a_{4} w= & \left(b_{1} w^{4}-b_{3} w^{2}+b_{5}\right) \sin \omega \tau \\
& +\left(b_{2} w^{3}-b_{4} w\right) \cos \omega \tau
\end{aligned}
$$




$$
\begin{aligned}
a_{1} w^{4}-a_{3} w^{2}+a_{5}= & -\left(b_{1} w^{4}-b_{3} w^{2}+b_{5}\right) \cos \omega \tau \\
& +\left(b_{2} w^{3}-b_{4} w\right) \sin \omega \tau
\end{aligned}
$$

Squaring and adding the two equations of (28), we have

$$
\omega^{10}+c_{1} \omega^{8}+c_{2} \omega^{6}+c_{3} \omega^{4}+c_{4} \omega^{2}+c_{5}=0
$$

where

$$
\begin{aligned}
& c_{1}=a_{1}^{2}-2 a_{2}-b_{1}^{2}, \\
& c_{2}=a_{2}^{2}+2 a_{4}-2 a_{1} a_{3}+2 b_{1} b_{3}-b_{2}^{2}, \\
& c_{3}=a_{3}^{2}-2 a_{2} a_{4}-b_{3}^{2}+2 b_{2} b_{4}+2 a_{1} a_{5}-2 b_{1} b_{5}, \\
& c_{4}=a_{4}^{2}-b_{4}^{2}-2 a_{3} a_{5}+2 b_{3} b_{5}, \\
& c_{5}=a_{5}^{2}-b_{5}^{2} .
\end{aligned}
$$

Letting $z=\omega^{2}$ we obtain

$$
h(z)=z^{5}+c_{1} z^{4}+c_{2} z^{3}+c_{3} z^{2}+c_{4} z+c_{5}=0 .
$$

Denote

$$
\begin{aligned}
p_{1} & =-\frac{6}{25} c_{1}^{2}+\frac{3}{5} c_{2}, \\
q_{1} & =\frac{8}{125} c_{1}^{3}+\frac{6}{25} c_{1} c_{2}+\frac{2}{5} c_{3}, \\
r_{1} & =-\frac{3}{625} c_{1}^{4}+\frac{3}{125} c_{1}^{2} c_{2}-\frac{2}{25} c_{1} c_{3}+\frac{1}{5} c_{4}, \\
p_{2} & =-\frac{1}{3} p_{1}^{2}-4 r_{1}, \\
q_{2} & =-\frac{2}{27} p_{1}^{3}+\frac{8}{3} p_{1} r_{2}-q_{1}^{2}, \\
\Delta_{1} & =\frac{1}{27} p_{2}^{3}+\frac{1}{4} q_{2}^{2}, \\
s_{*} & =\sqrt[3]{-\frac{q_{2}}{2}+\sqrt{\Delta_{1}}+\sqrt[3]{-\frac{q_{2}}{2}}-\sqrt{\Delta_{1}}}+\frac{1}{3} p_{1}, \\
\Delta_{2} & =-s_{*}-p_{1}+\frac{2 q_{1}}{\sqrt{s_{*}-p_{1}}}, \\
\Delta_{3} & =-s_{*}-p_{1}-\frac{2 q_{1}}{\sqrt{s_{*}-p_{1}}}, \\
2\left(p_{1}-s_{*}\right) & \frac{1}{5} p .
\end{aligned}
$$

Suppose that (31) has positive roots $z_{k}, k=1,2,3,4,5$, where $w_{k}=\sqrt{z_{k}}$. From (28), we have

$$
\cos \omega_{k} \tau=\frac{\left(w^{5}-a_{2} w^{3}+a_{4} w\right)\left(b_{2} w^{3}-b_{4} w\right)-\left(a_{1} w^{4}-a_{3} w^{2}+a_{5}\right)\left(b_{1} w^{4}-b_{3} w^{2}+b_{5}\right)}{\left(b_{2} w^{3}-b_{4} w\right)^{2}+\left(b_{1} w^{4}-b_{3} w^{2}+b_{5}\right)^{2}}=L\left(\omega_{k}\right) .
$$

Therefore

$$
\begin{aligned}
\tau_{j}^{k}=\frac{1}{\omega_{k}}\left[\arccos L\left(\omega_{k}\right)\right. & +2 j \pi], \\
& k=1,2,3,4,5, j=0,1,2, \ldots .
\end{aligned}
$$

Then $\pm i w_{k}$ are a pair of purely imaginary roots of (26) with $\tau=\tau_{j}^{k}$.

Define

$$
\begin{aligned}
\tau_{0} & =\tau_{j_{0}}^{k_{0}}=\min _{1 \leq k \leq 5, j \geq 1}\left\{\tau_{j}^{k}\right\}, \\
w_{0} & =w_{k_{0}} .
\end{aligned}
$$

From Theorem 4 and by a similar argument as that in [21, 22], we have the following results.

Lemma 5. Suppose that $R_{1}>1$ and (22) hold.

(i) If one of the following holds: (a) $c_{5}<0$; (b) $c_{5} \geq 0$, $q_{1}=0, \Delta_{0} \geq 0$, and $p_{1}<0$ or $r_{1} \leq 0$ and there exist $z^{*} \in\left\{z_{1}, z_{2}, z_{3}, z_{4}\right\}$ such that $z^{*}>0$ and $h\left(z^{*}\right) \leq 0$; (c) $c_{5} \geq 0, q_{1} \neq 0, s_{*}>p_{1}, \Delta_{2} \geq 0$, or $\Delta_{3} \geq 0$ and there exist $z^{*} \in\left\{z_{1}, z_{2}, z_{3}, z_{4}\right\}$ such that $z^{*}>0$ and $h\left(z^{*}\right) \leq 0$; (d) $c_{5} \geq 0, q_{1} \neq 0, s_{*}<p_{1}$, $q_{1}^{2} / 4\left(p_{1}-s^{*}\right)^{2}+(1 / 2) s_{*}=0, \bar{z}>0$, and $h(\bar{z}) \leq 0$, then all the roots of (26) have negative real parts when $\tau \in\left[0, \tau_{0}\right)$.

(ii) If all the conditions (a)-(d) of (i) are not satisfied, then all roots of (26) have negative real parts for all $\tau \geq 0$.

We consider $\xi(\tau)=\xi(\tau)+i \omega(\tau)$ to be a root of (26) satisfying $\xi(\tau)=0$ and $\omega(\tau)=\omega_{0}$. Differentiating the two sides of (26) with respect to $\tau$ and noticing that $\xi$ is a function of $\tau$, then

$$
\begin{aligned}
\left(\frac{d \xi}{d \tau}\right)^{-1}= & -\frac{5 \xi^{4}+4 a_{1} \xi^{3}+3 a_{2} \xi^{2}+2 a_{3} \xi+a_{4}}{\xi\left(\xi^{5}+a_{1} \xi^{4}+a_{2} \xi^{3}+a_{3} \xi^{2}+a_{4} \xi+a_{5}\right)} \\
& +\frac{4 b_{1} \xi^{3}+3 b_{2} \xi^{2}+2 b_{3} \xi^{1}+b_{4}}{\xi\left(b_{1} \xi^{4}+b_{2} \xi^{3}+b_{3} \xi^{2}+b_{4} \xi+b_{5}\right)}-\frac{\tau}{\xi}
\end{aligned}
$$


From (28) we obtain

$$
\begin{aligned}
& {\left[\frac{d \operatorname{Re}(\xi(\tau))}{d \tau}\right]_{\tau=\tau_{k}^{j}}^{-1}} \\
& =-\frac{\left(5 \omega_{k}^{4}-3 a_{2} \omega_{k}^{2}+a_{4}\right)\left(-\omega_{k}^{6}+a_{2} \omega_{k}^{4}-a_{4} \omega_{k}^{2}\right)}{\left(-\omega_{k}^{6}+a_{2} \omega_{k}^{4}-a_{4} \omega_{k}^{2}\right)^{2}+\left(a_{1} \omega_{k}^{5}-a_{3} \omega_{k}^{3}+a_{5} \omega_{k}\right)^{2}} \\
& +\frac{\left(4 a_{1} \omega_{k}^{3}-2 a_{3} \omega_{k}\right)\left(a_{1} \omega_{k}^{5}-a_{3} \omega_{k}^{3}+a_{5} \omega_{k}\right)}{\left(-\omega_{k}^{6}+a_{2} \omega_{k}^{4}-a_{4} \omega_{k}^{2}\right)^{2}+\left(a_{1} \omega_{k}^{5}-a_{3} \omega_{k}^{3}+a_{5} \omega_{k}\right)^{2}} \\
& +\frac{\left(-3 b_{2} \omega_{k}^{2}+b_{4}\right)\left(b_{2} \omega_{k}^{4}-b_{4} \omega_{k}^{2}\right)}{\left(b_{2} \omega_{k}^{4}-b_{4} \omega_{k}^{2}\right)^{2}+\left(b_{1} \omega_{k}^{5}-b_{3} \omega_{k}^{3}+b_{5} \omega_{k}\right)^{2}} \\
& +\frac{\left(-4 b_{1} \omega_{k}^{3}+2 b_{3} \omega_{k}\right)\left(b_{1} \omega_{k}^{5}-b_{3} \omega_{k}^{3}+b_{5} \omega_{k}\right)}{\left(b_{2} \omega_{k}^{4}-b_{4} \omega_{k}^{2}\right)^{2}+\left(b_{1} \omega_{k}^{5}-b_{3} \omega_{k}^{3}+b_{5} \omega_{k}\right)^{2}}
\end{aligned}
$$

By (26) we get

$$
\begin{gathered}
\left(\omega^{5}-a_{2} \omega^{3}+a_{4} \omega\right)^{2}+\left(a_{1} \omega^{4}-a_{3} \omega^{2}+a_{5}\right)^{2} \\
=\left(b_{2} \omega^{3}-b_{4} \omega\right)^{2}+\left(b_{1} \omega^{4}-b_{3} \omega^{2}+b_{5}\right) .
\end{gathered}
$$

Then

$$
\begin{aligned}
& {\left[\frac{d \operatorname{Re}(\xi(\tau))}{d \tau}\right]_{\tau=\tau_{k}^{j}}^{-1}} \\
& \quad=\frac{5 z_{k}^{4}+4 c_{1} z_{k}^{3}+3 c_{2} z_{k}^{2}+2 c_{3} z_{k}+a_{4}}{\left(b_{1} \omega_{k}^{4}-b_{3} \omega_{k}^{2}+b_{5}\right)^{2}+\left(b_{2} \omega_{k}^{2}-b_{4}\right)^{2}} \\
& =\frac{h^{\prime}\left(z_{k}\right)}{\left(b_{1} \omega_{k}^{4}-b_{3} \omega_{k}^{2}+b_{5}\right)^{2}+\left(b_{2} \omega_{k}^{2}-b_{4}\right)^{2}} .
\end{aligned}
$$

Therefore, it follows that

$$
\begin{aligned}
& \operatorname{sign}\left[\frac{d \operatorname{Re}(\xi(\tau))}{d \tau}\right]_{\tau=\tau_{k}^{j}}=\operatorname{sign}\left[\frac{d \operatorname{Re}(\xi(\tau))}{d \tau}\right]_{\tau=\tau_{k}^{j}}^{-1} \\
& =\operatorname{sign}\left[h^{\prime}\left(z_{k}\right)\right] .
\end{aligned}
$$

Since $z_{k}>0$, then $\operatorname{Re}\left[d \xi_{k}(\tau) / d \tau\right]_{\tau=\tau_{k}^{j}}$ and $h^{\prime}\left(z_{k}\right)$ have the same sign.

From the above analysis and the Hopf bifurcation theorem for functional differential equation [20], we have the following result.

\section{Theorem 6. Suppose that $R_{1}>1$ and (22) hold.}

(i) If the conditions (a)-(d) of Lemma 5 are all not satisfied, then the chronic infection equilibrium $Q_{2}$ is locally asymptotically stable for all time delay $\tau \geq 0$.

(ii) If one of the conditions (a)-(d) of Lemma 5 is satisfied, then the chronic infection equilibrium $Q_{2}$ is locally asymptotically stable for $\tau \in\left[0, \tau_{0}\right)$.

(iii) If the condition of (ii) is satisfied and $h^{\prime}\left(z_{k}\right) \neq 0$, then system (2) undergoes a Hopfbifurcation at $Q_{2}$ when $\tau=$ $\tau_{0}$.

\section{Conclusion}

In this paper, we have studied an HIV infection model including infected cells in eclipse stage and delay in the activation of CTL immune response. The model is governed by reaction diffusion equations and the transmission process is modeled by a specific nonlinear incidence rate that includes many types of special incidence functions as special cases. First, we discussed the nonnegativity and boundedness of solutions and the existence of equilibria of system (2). The global stability of the infection-free equilibrium $Q_{0}$ has been given by the Lyapunov's direct method and LaSalle's invariance principal when the basic reproductive number $R_{0} \leq 1$, which means that the infection is cleared and the virus dies out. We also obtained the global asymptotic stability of the immunefree infection equilibrium when $R_{0}>1$ and condition (14) is satisfied, which means that the infection will become chronic without persistent CTL immune response. If $R_{0}>1$ and $R_{1}>1$, there exists a chronic infection equilibrium with CTL immune response $Q_{2}$. We have shown that the chronic infection equilibrium $Q_{2}$ is locally asymptotically stable when the delay is sufficiently small, but with the increase of the time delay, the stability of $Q_{2}$ may destabilize and lead to Hopf bifurcation.

The results of this paper reflect the fact that the immunological delay in (2) do not affect the positivity and boundedness of solutions and the global stability of the infection-free equilibrium and immune-free equilibrium. For the chronic infection equilibrium, in the absence of delay, the globally stability is obtained, a small delay does not affect the local stability, and Hopf bifurcation may occur when the time delay is large enough.

\section{Conflicts of Interest}

The authors declare that they have no conflicts of interest regarding the publication of this paper.

\section{References}

[1] WHO, "HIV/AIDS, July 2017," http://www.who.int/\%20mediacentre/factsheets/fs360/en/.

[2] M. A. Nowak and C. R. M. Bangham, "Population dynamics of immune responses to persistent viruses," Science, vol. 272, no. 5258, pp. 74-79, 1996.

[3] L. Rong, M. A. Gilchrist, Z. Feng, and A. S. Perelson, "Modeling within-host HIV-1 dynamics and the evolution of drug resistance: trade-offs between viral enzyme function and drug susceptibility," Journal of Theoretical Biology, vol. 247, no. 4, pp. 804-818, 2007.

[4] Z. Hu, W. Pang, F. Liao, and W. Ma, "Analysis of a cd4+t cell viral infection model with a class of saturated infection rate," Discrete and Continuous Dynamical Systems - Series B, vol. 19, no. 3, pp. 735-745, 2014.

[5] M. Maziane, E. M. Lotfi, K. Hattaf, and N. Yousfi, "Dynamics of a Class of HIV Infection Models with Cure of Infected Cells in Eclipse Stage," Acta Biotheoretica, vol. 63, no. 4, pp. 363-380, 2015.

[6] K. Hattaf, N. Yousfi, and A. Tridane, "Stability analysis of a virus dynamics model with general incidence rate and two delays," 
Applied Mathematics and Computation, vol. 221, pp. 514-521, 2013.

[7] J. R. Beddington, "Mutual interference between parasites or predat ors and its effect on searching efficiency," Journal of Animal Ecology, vol. 44, pp. 331-340, 1975.

[8] D. L. DeAngelis, A. H. Goldstein, and R. V. O'Neill, "A model for trophic interaction," Ecology, vol. 56, pp. 881-892, 1975.

[9] P. Crowley and E. Martin, "Functional responses and interference within and between year classes of a dragonfly population," Journal of the North American Benthological Society, vol. 8, pp. 211-221, 1989.

[10] C. Lv, L. Huang, and Z. Yuan, "Global stability for an HIV-1 infection model with Beddington-DeAngelis incidence rate and CTL immune response," Communications in Nonlinear Science and Numerical Simulation, vol. 19, no. 1, pp. 121-127, 2014.

[11] M. Maziane, K. Hattaf, and N. Yousfi, "Global stability for a class of HIV infection models with cure of infected cells in eclipse stage and CTL immune response," International Journal of Dynamics and Control, 2016.

[12] C. C. Travis and G. F. Webb, "Existence and stability for partial functional differential equations," Transactions of the American Mathematical Society, vol. 200, pp. 395-418, 1974.

[13] J. Wu, Theory and Applications of Partial Functional-Differential Equations, Springer, New York, NY, USA, 1996.

[14] W. E. Fitzgibbon, "Semilinear functional differential equations in Banach space," Journal of Differential Equations, vol. 29, no. 1, pp. 1-14, 1978.

[15] J. Martin and H. L. Smith, "Abstract functional-differential equations and reaction-diffusion systems," Transactions of the American Mathematical Society, vol. 321, no. 1, pp. 1-44, 1990.

[16] R. H. Martin and H. L. Smith, "Reaction-diffusion systems with time delays: Monotonicity, invariance, comparison and convergence," Journal für die reine und Angewandte Mathematik, vol. 1991, no. 413, pp. 1-35, 1991.

[17] M. H. Protter and H. F. Weinberger, Maximum Principles in Differential Equations, Prentice-Hall, Englewood Cliffs, NJ, USA, 1967.

[18] D. Henry, Geometric Theory of Semilinear Parabolic Equations, vol. 840 of Lecture Notes in Mathematics, Springer, New York, NY, USA, 1993.

[19] K. Hattaf and N. Yousfi, "Global stability for reaction-diffusion equations in biology," Computers \& Mathematics with Applications, vol. 66, no. 8, pp. 1488-1497, 2013.

[20] J. K. Hale and S. M. Verduyn Lunel, Introduction to FunctionalDifferential Equations, Springer, Berlin, Germany, 1993.

[21] M. Maziane, E. Lotfi, K. Hattaf, and N. Yousfi, "Impact of Delay in Immune Response Activation on HIV Infection Dynamics," British Journal of Mathematics \& Computer Science, vol. 21, no. 4, pp. 1-15, 2017.

[22] T. Zhang, H. Jiang, and Z. Teng, "On the distribution of the roots of a fifth degree exponential polynomial with application to a delayed neural network model," Neurocomputing, vol. 72, no. 46, pp. 1098-1104, 2009. 


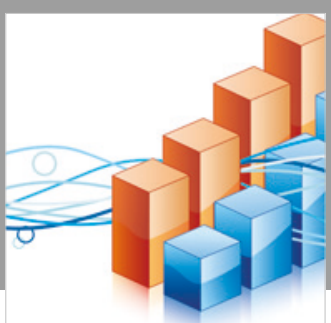

Advances in

Operations Research

\section{-n-m}
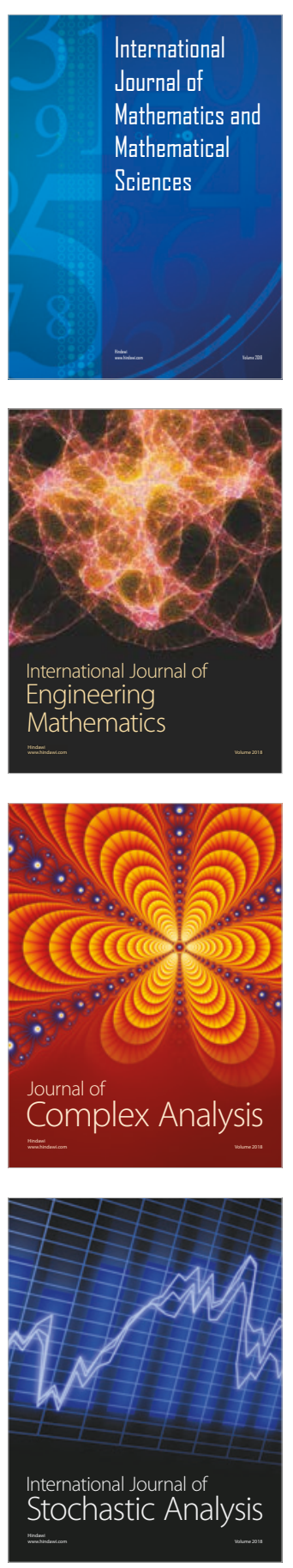
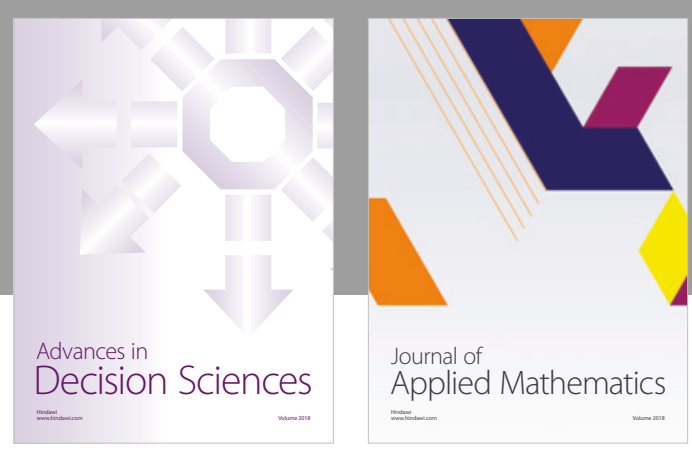

Journal of

Applied Mathematics
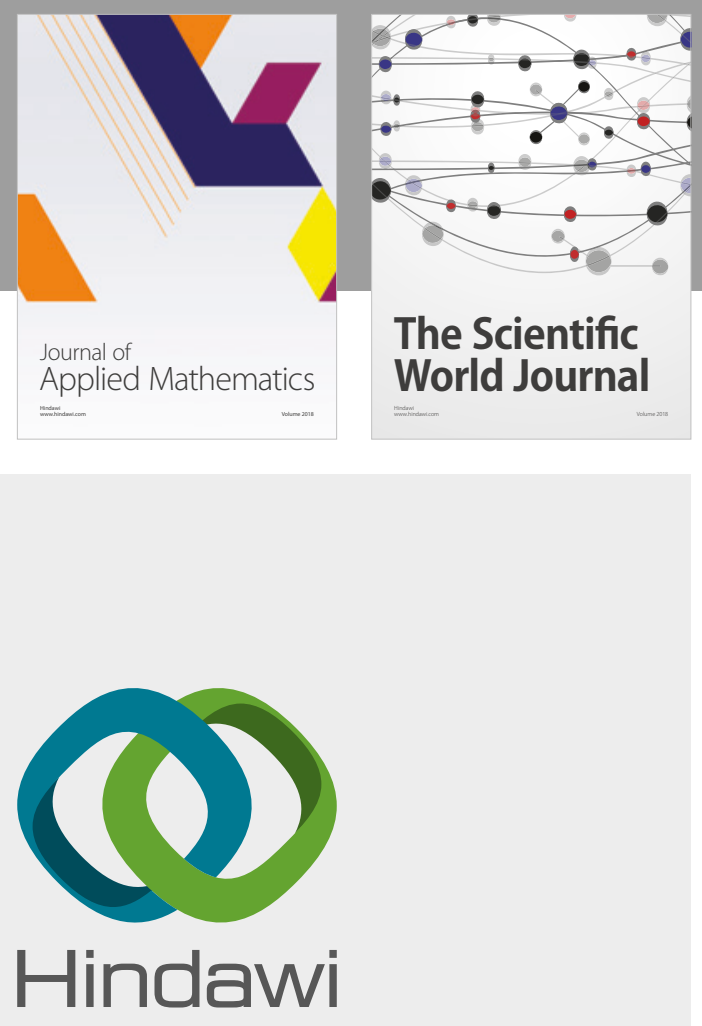

Submit your manuscripts at

www.hindawi.com

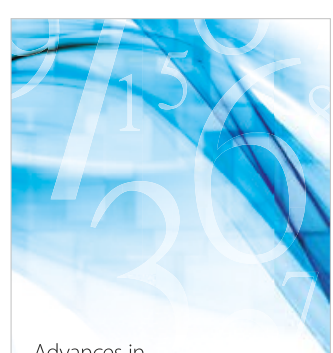

Advances in
Numerical Analysis
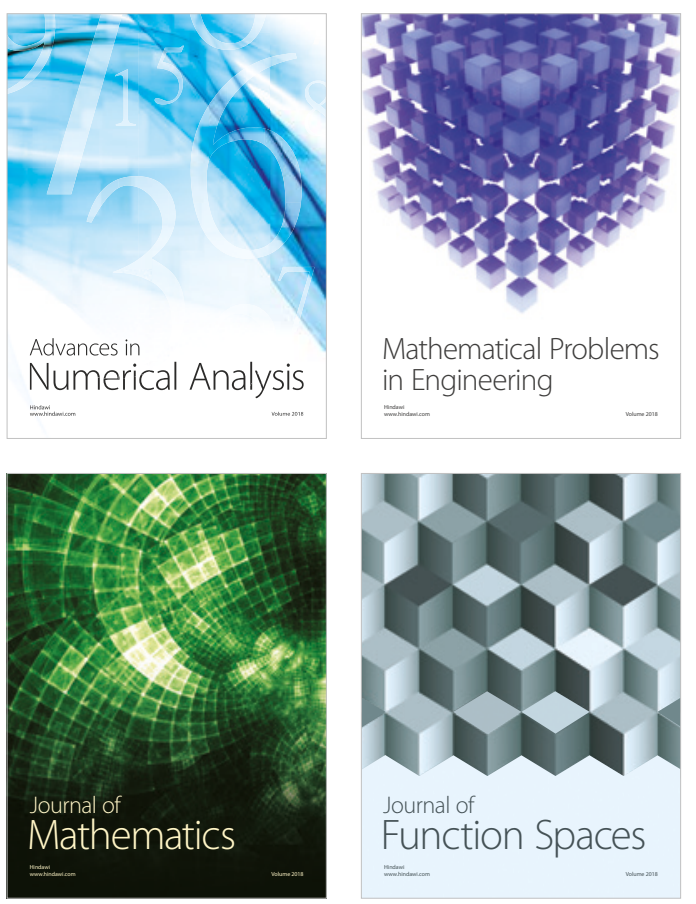

Mathematical Problems in Engineering

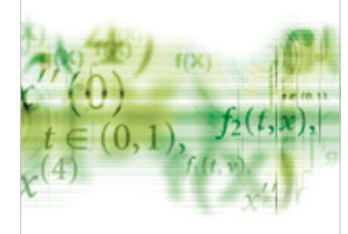

International Journal of

Differential Equations

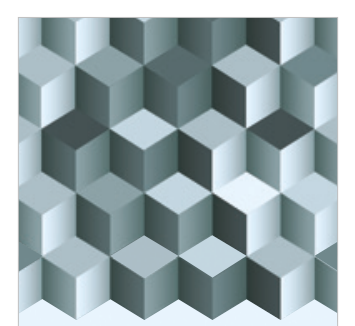

Journal of

Function Spaces

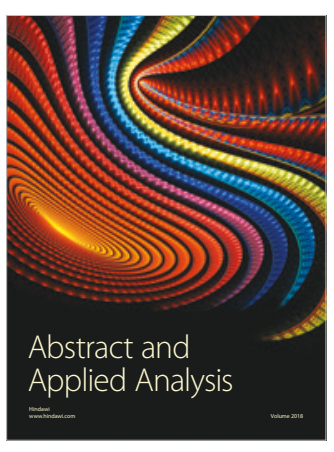

The Scientific

World Journal

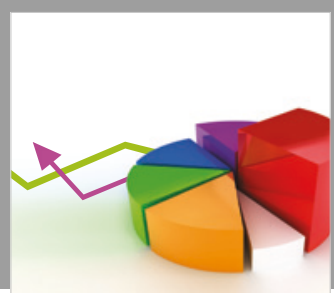

Journal of

Probability and Statistics
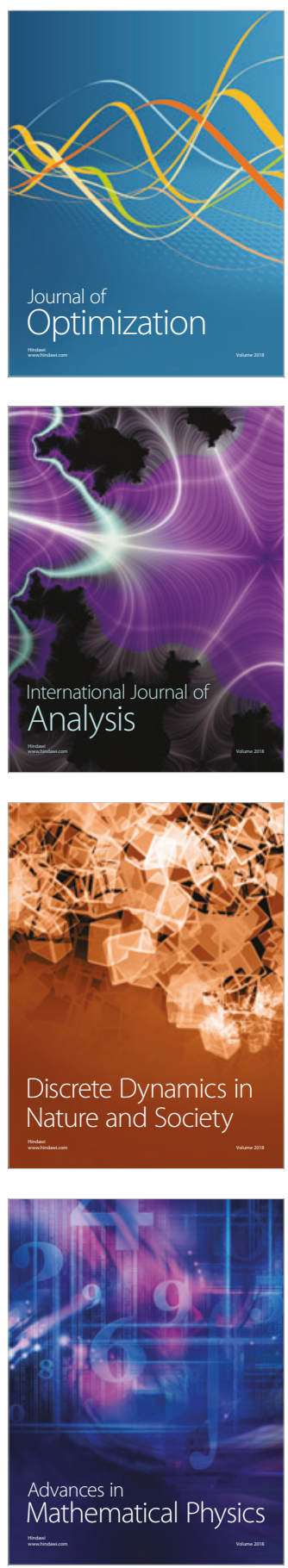